\title{
DNA integrity index as a potential molecular biomarker in colorectal cancer
}

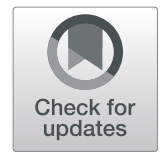

\author{
Ramy Salem ${ }^{1 *}$ (D), Rasha Ahmed ${ }^{1}$, Karim Shaheen ${ }^{1}$, Mohammed Abdalmegeed $^{2}$ and Heba Hassan ${ }^{1}$
}

\begin{abstract}
Background: Efficient approaches for early detection of colorectal cancer offer opportunities to gain better treatment outcomes. Blood-based molecular biomarkers as DNA integrity index (DII) might represent a promising tumor marker in the future. The purpose of this study was to assess the clinical utility of the DII as a potential biomarker for colorectal cancer in 90 colorectal cancer patients, 30 patients with benign colorectal mass, and 30 age- and sex-matched healthy control subjects. PCR was used to assess the concentration of both ALU115 and ALU247. DII was calculated as the ratio of Q247/Q115.

Results: DII was significantly higher in colorectal cancer patients than both patients with benign colorectal mass and healthy controls. ROC curve was plotted using DII and the best cut-off was $\geq 0.60$ with diagnostic sensitivity 93.0\%, specificity 65.0\%, PPV 80.0\%, NPV 86.0\%, and efficiency 82\% with AUC (0.872) while the best cut-off for CEA was $\geq 1.4 \mathrm{ng} / \mathrm{mL}$ with diagnostic sensitivity $87.0 \%$, specificity $60.0 \%$, PPV $76 \%$, NPV $75 \%$, and efficiency $76 \%$ with AUC (0.79).
\end{abstract}

Conclusions: Our results suggest that DII is better than CEA as an early marker for colorectal cancer detection and may be used as a candidate biomarker for malignancy.

Keywords: Colorectal cancer, DII, ALU

\section{Background}

Colorectal $(\mathrm{CRC})$ is ranked the third most frequent malignancy in men after lung and prostate tumors, and the second most commonly diagnosed malignancy in women after breast cancer and is considered the second leading cause of cancer mortalities [1]. The 5-year survival rate for CRC patients relies on the staging of the disease at diagnosis. Therefore, it is important to diagnose and manage CRC at the early stages to improve treatment outcomes and decrease cancer-related mortality [2].

Current screening approaches include stool-based tests and colonoscopy. Stool-based tests as fecal occult blood testing (FOBT) are cheap and non-invasive but with low sensitivity. Colonoscopy-guided biopsy is

\footnotetext{
* Correspondence: ramysalem@med.asu.edu.eg

${ }^{1}$ Clinical Pathology Department, Faculty of Medicine, Ain Shams University, Cairo, Egypt

Full list of author information is available at the end of the article
}

the gold-standard screening and diagnostic approach with high specificity up to more than $95 \%$, but it is invasive and necessitates bowel preparation and also may cause severe complications [3, 4]. Conventional tumor markers, as carcinoembryonic antigen (CEA) and carbohydrate antigen 19.9 (CA 19.9), have limited sensitivity and specificity limiting their efficacy in screening and diagnosis. Nevertheless, they are clinically used to detect prognosis, monitor disease progression, and response to treatment [5]. Since the conventional methods for CRC screening are either inefficient or invasive, there is a high need to offer more compliant and less-invasive screening methods with high sensitivity and specificity.

Cell-free DNA (CfDNA) has been suggested to be a promising tumor marker. However, its level is also elevated in various non-malignant disorders. Therefore, more specific approaches such as measuring the integrity of DNA have been proposed $[6,7]$. This approach is 
based on the difference in length of cfDNA released from the cells according to the mechanism of cell death. Apoptosis, which usually occurs in normal tissues, results in short DNA fragments less than 180 bp, while necrosis, which occurs in tumor cells, produces longer fragments. The Arthrobacter luteus (ALU) repeats are $300 \mathrm{bp}$ in length and are the most predominant repeated sequences in the human genome, with a copy number of $1.4 \times 10^{6}$ per genome. It accounts for more than $10 \%$ of the human genome [8]. Most studies used ALU quantitative polymerase chain reaction (PCR) for calculation of DNA integrity index (DII) that represents the ratio between ALU247 long fragments released from necrotic cells and ALU115 short fragments released from normal cells [4]. This study aimed to assess the clinical utility of DII as a potential biomarker for CRC and to evaluate its correlation with the traditional tumor marker (CEA).

\section{Methods}

The study included 90 adult CRC patients, 30 patients with benign colorectal mass, and 30 age- and sexmatched healthy controls. CRC patients who previously received chemotherapy/radiotherapy or undergone colorectal surgery as well as those who have other types of malignant tumors or a history of autoimmune diseases were excluded from this study. CRC and benign mass diagnosis were done using colonoscopy and biopsy followed by histopathological diagnosis. Magnetic resonance imaging (MRI) anorectal protocol and /or Pancomputerized tomography (CT) were done for CRC patients to detect metastasis. Written informed consent was obtained from each participant prior to participation in the study. Demographic and clinical characteristics of participants are shown in Table 1.

\section{Sample collection}

Three milliliters of whole blood were collected into a sterile plain vacutainer for PCR analysis. Double centrifugation was done within $4 \mathrm{~h}$ of collection. The first centrifugation was at $1600 \times g$ for $10 \mathrm{~min}$, and the resultant supernatant was carefully harvested into another plain sterile tube and subjected to second centrifugation at $16000 \times g$ for $10 \mathrm{~min}$. Serum was stored frozen at $-80{ }^{\circ} \mathrm{C}$ until used for PCR. Another $3 \mathrm{~mL}$ of blood was collected into a plain vacutainer tube and centrifuged at $3000 \times g$ for $20 \mathrm{~min}$ and serum was used for the assay of CEA.

\section{Assessment of CEA levels}

CEA levels were measured using Cobas e411 immunoassay autoanalyzer (Roche Diagnostics, D-68305 Mannheim).

\section{DNA extraction and ALU qPCR:}

Deoxyribonucleic acid (DNA) extraction was performed from serum using QIAamp DNA blood mini kit (QIAG EN, Germany). Extracted DNA concentration was determined by measuring the absorbance at $260 \mathrm{~nm}$ using spectrophotometer. Meanwhile, the ratio of the absorbance at 260 and $280 \mathrm{~nm}$ (OD260/OD280) was used to assess the purity of extracted DNA. A ratio of about 1.8 was generally accepted.

Two PCR reactions were set for each sample. ALU 115 primers were used for the first reaction, and ALU 247 primers were used for the second reaction. The sequence of used primers are shown in Table 2.

Reaction volume was set as follows: $10 \mu \mathrm{L}$ of the Maxima SYBR Green qPCR Master Mix, $0.6 \mu \mathrm{L}$ of forward primer, $0.6 \mu \mathrm{L}$ of reverse primer, $3.8 \mu \mathrm{L}$ of RNASE free water, and $5 \mu \mathrm{L}$ of DNA extract. PCR was performed on Applied Biosystem Step One Real-Time PCR System. Amplification was performed according to the following protocol: initial heat activation at $95{ }^{\circ} \mathrm{C}$ for $10 \mathrm{~min}$, DNA denaturation at $95^{\circ} \mathrm{C}$ for $30 \mathrm{~s}$ followed by annealing at $64{ }^{\circ} \mathrm{C}$ for $30 \mathrm{~s}$ and extension at $72{ }^{\circ} \mathrm{C}$ for $30 \mathrm{~s}$ for 40 cycles. PCR amplification was followed by melting curve analysis and gel electrophoresis. Calibration curves were constructed using Taqman Control Genomic Human DNA (Applied Biosystems, Thermofisher, USA) for the calculation of the concentration of both ALU115 and ALU247. Finally, DII was calculated as the ratio of Q247/Q115.

\section{Statistical analysis}

Data analysis was done using IBM SPSS statistics (V. 22.0, IBM Corp., USA, 2013). Data were presented as median and interquartile range for non-parametric data, mean, and standard deviation for parametric data, frequency, and percentage for qualitative data. Groups were compared using Kruskal-Wallis test then post hoc "Dunn's multiple comparison test" was used for pairwise comparison. Spearman's rank correlation coefficient $\left(r_{\mathrm{s}}\right)$ was used to test the correlation between numerical variables. The receiver operating characteristic (ROC) curve was plotted for diagnostic test evaluation. A $p$ value $<0.05$ was considered significant.

\section{Results \\ Higher DII and ALU115, ALU 247, and CEA levels in CRC patients than controls}

A significant difference was found between the three studied groups regarding ALU $115(p \leq 0.01)$, ALU 247 $(p \leq 0.01)$, DII $(p \leq 0.01)$, and CEA $(p=0.002)$. Post hoc analysis revealed higher levels of ALU115 and ALU 247 in CRC patients than healthy controls $(p<0.001$ for both), also higher levels were observed in pathological controls than healthy controls $(p=0.001$ and $p=0.009$, 
Table 1 Demographic and clinical characteristics:

\begin{tabular}{|c|c|c|c|}
\hline Parameter & $\begin{array}{l}\text { CRC patients } \\
(n=90)\end{array}$ & $\begin{array}{l}\text { Pathological controls } \\
(n=30)\end{array}$ & $\begin{array}{l}\text { Healthy controls } \\
(n=30)\end{array}$ \\
\hline $\begin{array}{l}\text { Age (years) } \\
\text { mean } \pm \text { SD }\end{array}$ & $52 \pm 9.5$ & $50 \pm 4.7$ & $51 \pm 5.9$ \\
\hline $\begin{array}{l}\text { Sex } \\
\quad \text { Male (\%) } \\
\text { Female (\%) }\end{array}$ & $\begin{array}{l}48 / 90(53 \%) \\
42 / 90(47 \%)\end{array}$ & $\begin{array}{l}12 / 30(40 \%) \\
18 / 30(60 \%)\end{array}$ & $\begin{array}{l}21 / 30(70 \%) \\
9 / 30(30 \%)\end{array}$ \\
\hline $\begin{array}{l}\text { Anemia } \\
\quad \text { Present (\%) } \\
\text { Absent (\%) }\end{array}$ & $\begin{array}{l}66 / 90(73.3 \%) \\
24 / 90(36.7 \%)\end{array}$ & $\begin{array}{l}12 / 30(40 \%) \\
18 / 30(60 \%)\end{array}$ & $\begin{array}{l}0 / 30 \\
30 / 30(100 \%)\end{array}$ \\
\hline $\begin{array}{l}\text { Constipation } \\
\text { Present (\%) } \\
\text { Absent (\%) }\end{array}$ & $\begin{array}{l}57 / 90(63.3 \%) \\
33 / 90(36.7 \%)\end{array}$ & $\begin{array}{l}0 / 30 \\
30 / 30(100 \%)\end{array}$ & $\begin{array}{l}0 / 30 \\
30 / 30(100 \%)\end{array}$ \\
\hline $\begin{array}{l}\text { Bleeding per rectum } \\
\text { Present (\%) } \\
\text { Absent (\%) }\end{array}$ & $\begin{array}{l}51 / 90(56.7 \%) \\
39 / 90(43.3 \%)\end{array}$ & $\begin{array}{l}3 / 30(30 \%) \\
27 / 30(90 \%)\end{array}$ & $\begin{array}{l}0 / 30 \\
30 / 30(100 \%)\end{array}$ \\
\hline $\begin{array}{c}\text { Palpable mass } \\
\text { Present (\%) } \\
\text { Absent (\%) }\end{array}$ & $\begin{array}{l}3 / 90(3.3 \%) \\
87 / 90(96.7 \%)\end{array}$ & $\begin{array}{l}0 / 30 \\
30 / 30 \text { (100\%) }\end{array}$ & $\begin{array}{l}0 / 30 \\
30 / 30(100 \%)\end{array}$ \\
\hline $\begin{array}{l}\text { Family history } \\
\text { Present (\%) } \\
\text { Absent (\%) }\end{array}$ & $\begin{array}{l}21 / 90(20 \%) \\
69 / 90(80 \%)\end{array}$ & $\begin{array}{l}6 / 30(20 \%) \\
24 / 30(80 \%)\end{array}$ & $\begin{array}{l}0 / 30 \\
30 / 30(100 \%)\end{array}$ \\
\hline $\begin{array}{l}\text { Tumor size } \\
\text { (mean } \pm \text { SD) }\end{array}$ & $6.6 \pm 1.9$ & NA & NA \\
\hline $\begin{array}{l}\text { Site of tumor } \\
\text { Left (\%) } \\
\text { Right (\%) } \\
\text { Rectum (\%) }\end{array}$ & $\begin{array}{l}30 / 90(33.3 \%) \\
33 / 90(36.7 \%) \\
27 / 90(30 \%)\end{array}$ & NA & NA \\
\hline $\begin{array}{l}\text { Grade of tumor } \\
\quad 1(\%) \\
2(\%) \\
3(\%)\end{array}$ & $\begin{array}{l}9 / 90(10 \%) \\
78 / 90(86.7 \%) \\
3 / 90(3.3 \%)\end{array}$ & NA & NA \\
\hline $\begin{array}{l}\text { TNM stage } \\
1(\%) \\
2(\%) \\
3(\%) \\
4(\%)\end{array}$ & $\begin{array}{l}6 / 90(6.7 \%) \\
21 / 90(23.3 \%) \\
30 / 90(33.3 \%) \\
33 / 90(36.7 \%)\end{array}$ & NA & NA \\
\hline $\begin{array}{l}\text { Lymph nodes metastasis } \\
\text { Present (\%) } \\
\text { Absent (\%) }\end{array}$ & $\begin{array}{l}30 / 90(33.3 \%) \\
27 / 90(30 \%)\end{array}$ & NA & NA \\
\hline $\begin{array}{l}\text { Distant metastasis } \\
\text { Present (\%) } \\
\text { Absent (\%) }\end{array}$ & $\begin{array}{l}33 / 90(36.7 \%) \\
57 / 90(63.3 \%)\end{array}$ & NA & NA \\
\hline
\end{tabular}

CRC colorectal cancer, NA non-applicable, SD standard deviation, TNM tumor size, node involvement, and metastasis status

respectively) while no difference was observed between CRC patients and pathological controls $(p=0.65$ and $p$ $=0.66$, respectively) (Fig. $1 \mathrm{a}, \mathrm{b}$ ). Additionally, higher DII was observed in CRC patients than in both pathological

Table 2 Primers sequences

\begin{tabular}{ll}
\hline ALU 115 Forward primer $\left(F_{115}\right)$ & 5' CCTGAGGTCAGGAGTTCGAG'3 \\
ALU 115 Reverse primer $\left(R_{115}\right)$ & 5' CCCGAGTAGCTGGGATTACA'3 \\
ALU 247 Forward primer $\left(F_{247}\right)$ & 5' GTGGCTCACGCCTGTAATC'3 \\
ALU 247 Reverse primer $\left(R_{247}\right)$ & 5' CAGGCTGGAGTGCAGTGG'3.
\end{tabular}

$A L U$ the Arthrobacter luteus, $F$ forward, $R$ reverse controls $(p=0.037)$ and healthy controls $(p<0.001)$ while no difference was observed between pathological controls and healthy controls $(p=0.31)$ (Fig. 1c). Also, significantly higher levels of CEA were found in CRC patients compared to both pathological controls $(p=$ $0.038)$ and healthy controls $(p=0.009)$ while no difference was observed between pathological controls and healthy controls $(p=0.71)$ (Fig. 1d).

\section{DII and ALU 247 are elevated in early stages of CRC}

To examine the usefulness of the different parameters in the early diagnosis of cancer colon, we compared 


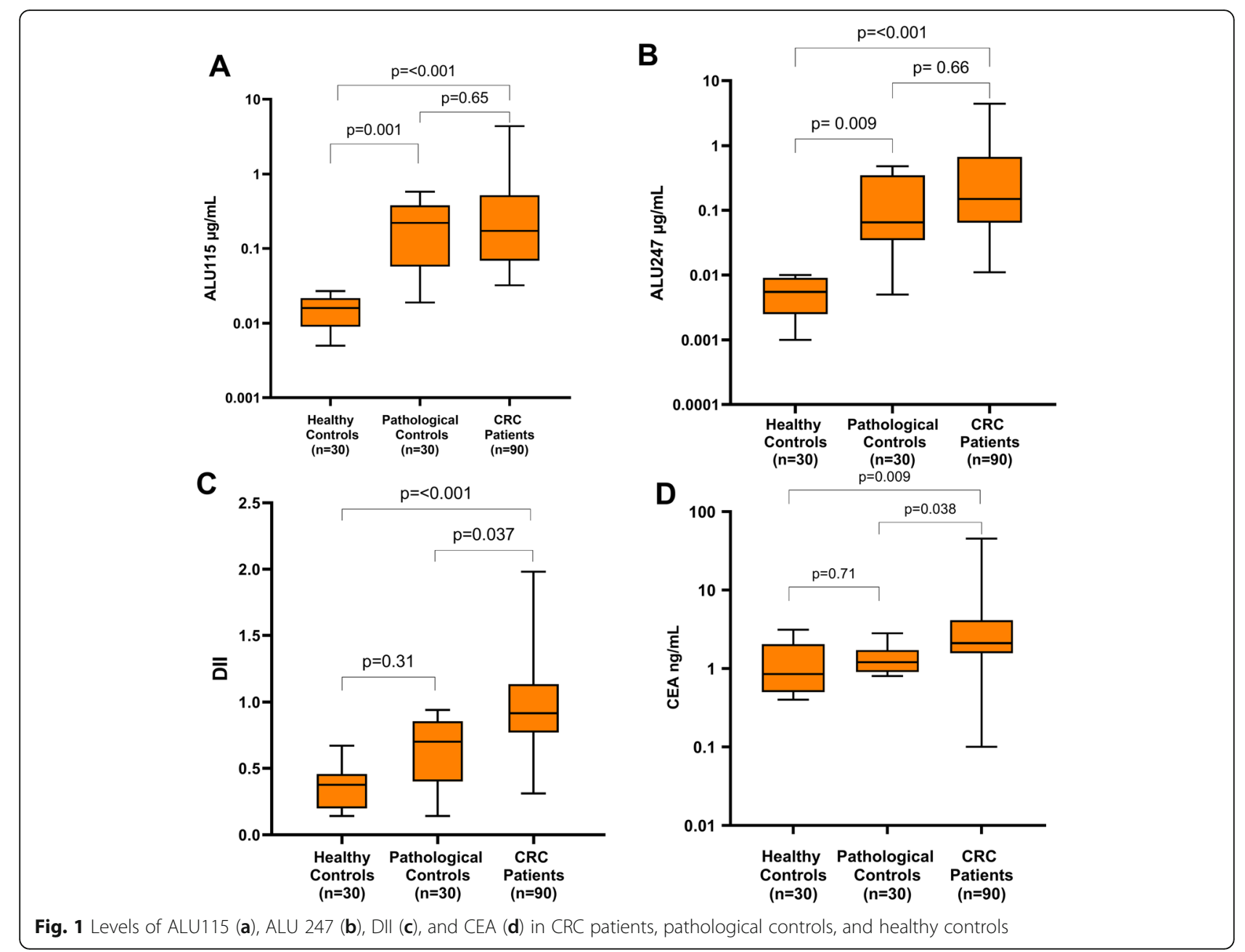

their levels in early stage CRC (stage I and II) to healthy controls. A significant higher DII and ALU 247 concentration in patients with early stages was observed compared to controls ( $p=0.01$ for both) while no difference was found regarding CEA and ALU115 concentrations $(p=0.25$ and $p=0.07$, respectively) (Fig. 2).

\section{Comparison of different parameters between metastatic and non-metastatic CRC}

To examine whether DII, ALU 115 levels, ALU 247 levels, and CEA levels are increased in metastatic cancer colon or not, we compared different parameters between CRC patients with metastasis and CRC patients with no metastasis. Only levels of DII and CEA were significantly higher in the metastatic CRC patients than the nonmetastatic patients $(p=0.004$ and $p=0.04$, respectively) while no difference was observed in ALU115 and ALU 247 ( $p=0.44$ and $p=0.49$ ) (Fig. 3).
DIl correlated with CEA, tumor size, and tumor stage Correlation study revealed significant positive correlation of DII with CEA $\left(r_{\mathrm{s}}=0.39, p=0.03\right)$ (Fig. 4a), tumor size $\left(r_{\mathrm{s}}=0.40, p=0.02\right)$ (Fig. $\left.4 \mathrm{~b}\right)$, and tumor stage $\left(r_{\mathrm{s}}=0.39, p=0.03\right)$ (Fig. 4c).

\section{Diagnostic performance of DII in discriminating between} CRC patients and both pathological and healthy controls ROC curve was plotted to determine the diagnostic performance of DII in discriminating between CRC patients and healthy controls. The best cut-off was 0.55 with a diagnostic sensitivity of $93.3 \%$, specificity of $90.0 \%$, positive predictive value (PPV) of $96.5 \%$, negative predictive value (NPV) of $81.8 \%$, and efficiency of $92.5 \%$. The area under the curve (AUC) was 0.95 with $95 \%$ CI of (0.89-1.0) (Fig. 5a).

Regarding the performance of DII in discriminating between CRC patients and pathological controls, the best cut-off was 0.66 with a diagnostic sensitivity of $86.6 \%$, specificity of $50.0 \%$, PPV of $85.0 \%$, NPV of $63.0 \%$, 
A

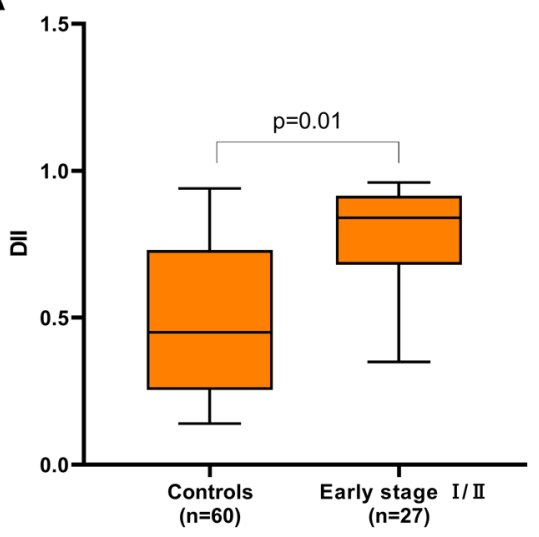

C

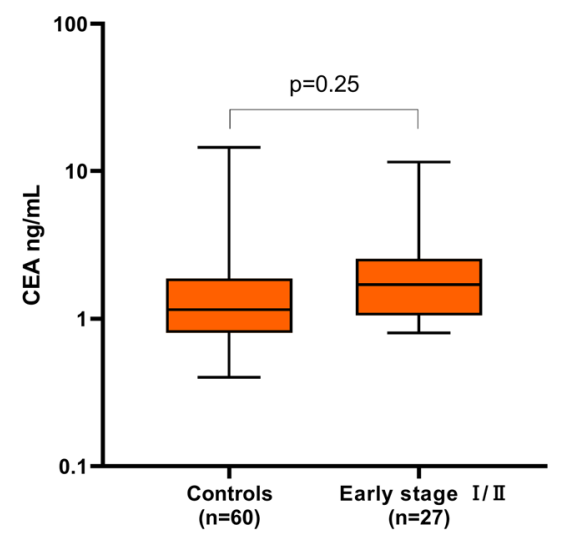

B

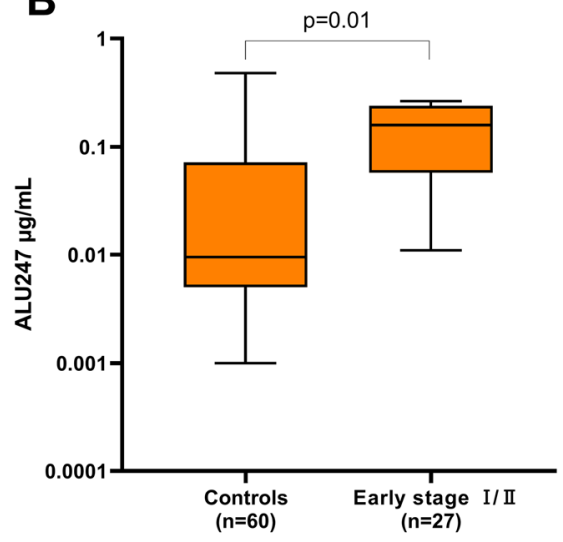

D

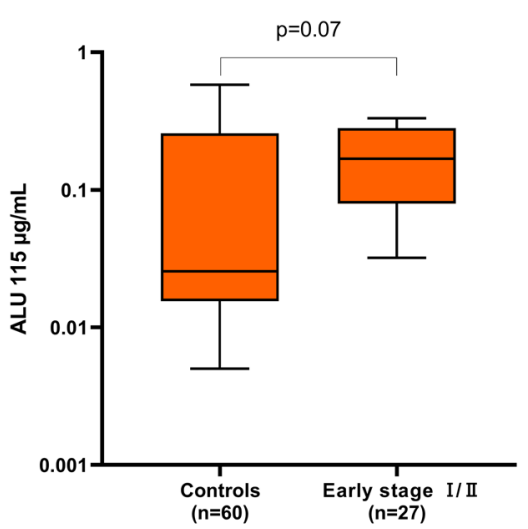

Fig. 2 Higher DII (a) and ALU 247 (b) levels in patients with early CRC stages while no difference was found regarding CEA (c) and ALU115 (d)

and efficiency of $80 \%$ with AUC of 0.792 and $95 \%$ CI (0.64-0.93) (Fig. 5b).

Another ROC curve was plotted for DII to discriminate CRC patients from all controls (both pathological and healthy), and the best cut-off was 0.60 with a diagnostic sensitivity of $93.0 \%$, specificity of $65.0 \%$, PPV of $80.0 \%$, NPV of $86.0 \%$, and efficiency of $82 \%$ with AUC of 0.872 and $95 \%$ CI (0.77-0.96) (Fig. 5c).

\section{Discussion}

Colorectal cancer remains a significant cause of morbidity and mortality worldwide with a high incidence rate. The discovery of non-invasive biomarkers for CRC detection with adequate sensitivity and specificity is a major challenge to reduce cancer-related morbidity and mortality. New non-invasive tests are under research to meet the balance between the increase of sensitivity and decreasing the need for unnecessary colonoscopies [9].

Circulating free DNA has been suggested to be a promising tumor marker. However, as cfDNA levels may also get elevated in various non-malignant disorders, more specific approaches have been proposed. Among these approaches is the calculation of the DII. The DII describes the ratio of longer free DNA fragments to shorter free DNA fragments [6, 7]. In healthy individuals, cfDNA mainly originates from apoptotic cells which usually release DNA fragments of 185-200 bp. In contrast, cfDNA released from cancer cells is usually longer due to the pathologic cell death in tumors. Therefore, DNA integrity has the potential of being used for tumor detection and prognostic prediction [4]. DII calculation using fragments from GAPDH has been described by Van Beers et al. [10], also Salvianti et al. [11] has investigated the determination of DII targeting sequences in amyloid precursor protein.

The use of ALU repeats for determination of DII was first proposed by Umetani et al. [12]. ALU115 and ALU247 are 2 amplicons with a length of 115 and 247 bp. ALU115 and ALU247 are used to distinguish between DNA originating from apoptotic and necrotic cell death, respectively. Since the main source of short cfDNA (180-200 bp fragments) in healthy individuals 
A

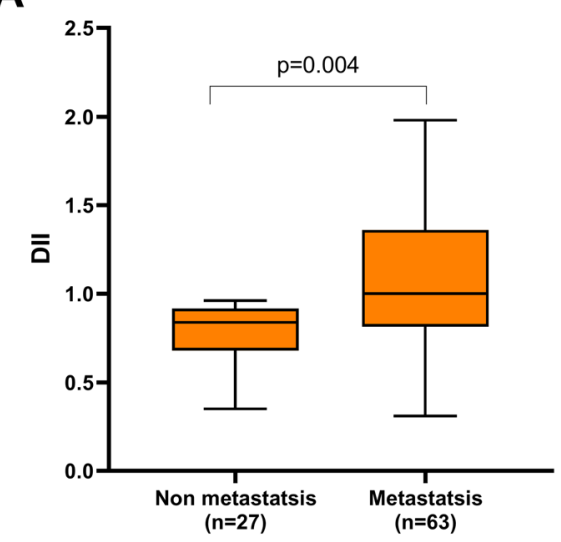

C

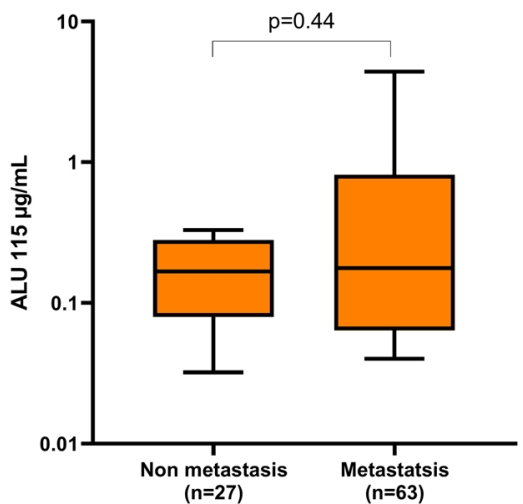

B

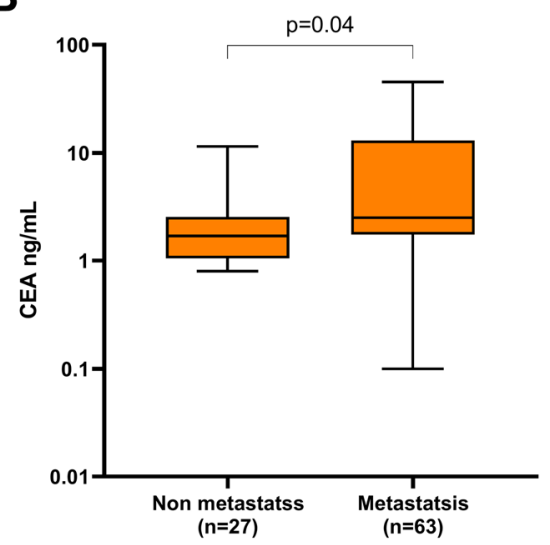

D

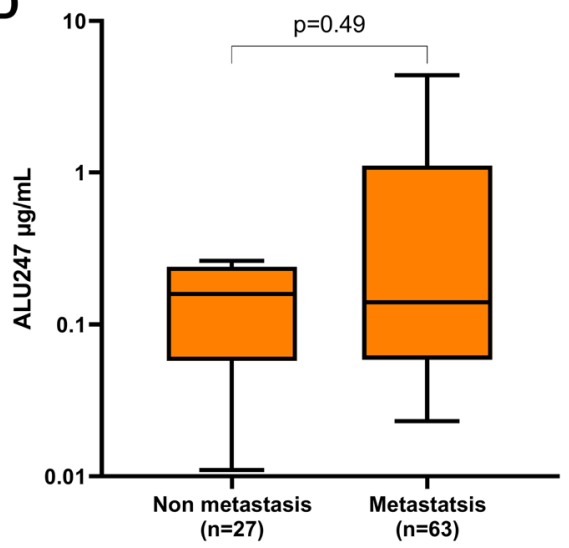

Fig. 3 Higher DII (a) and CEA (b) in metastatic CRC patients than in the non-metastatic patients while no difference was observed in ALU115 (c) and ALU 247 (d)

has been attributed to apoptotic cells, a majority of longer DNA fragments (ALU247) could represent a biomarker for malignant tumor detection. The annealing sites of ALU115 are within the ALU247 ones, the ALU115 primers could amplify both shorter (truncated by apoptosis, i.e., ALU115) and longer DNA fragments (ALU247), so results of ALU115 quantitation represent the total amount of cfDNA. However, ALU247 primers amplify only longer DNA fragments; therefore, results of ALU247 quantitation represent amounts of DNA released from necrotic cell death. DII is calculated as the ratio of longer to shorter ALU fragments (ALU247/ ALU115) [12].

In this study, we chose ALU115 and ALU247 repeats to calculate the DII in CRC patients. We aimed to assess the clinical utility of DII as a potential biomarker for CRC and to evaluate its correlation with CEA, the conventional marker used for prognosis, and follow-up of CRC patients.

We found higher DII in CRC patients than both pathological controls and healthy controls. DII was higher in early stage CRC patients compared to healthy controls and was also higher in the metastatic CRC patients compared to non-metastatic patients. Also, DII positively correlated with CEA, tumor size, and tumor stage.

Umetani et al. [12] investigated the DII in 32 CRC patients and 51 heathy controls in the USA and reported that DII was higher in CRC patients compared to healthy controls with an AUC of 0.78 for discriminating CRC patients from healthy individuals. Similarly, Leszinski et al. [6] assessed DII in 24. CRC patients, 11 patients with benign gastrointestinal diseases, and 24 healthy individuals in Germany, and reported higher DII in CRC patients compared to healthy controls with an AUC of 0.738 while no difference was observed between CRC patients and patients with benign gastrointestinal diseases. El-Gayar et al. [13] also assessed DII in 50 CRC patients and 20 healthy controls and reported that DII is higher in CRC patients compared to healthy individuals with AUC of 0.9.

As expected, the level of CEA was significantly higher in CRC patients than in both the healthy control group and the pathological control group. This came in 


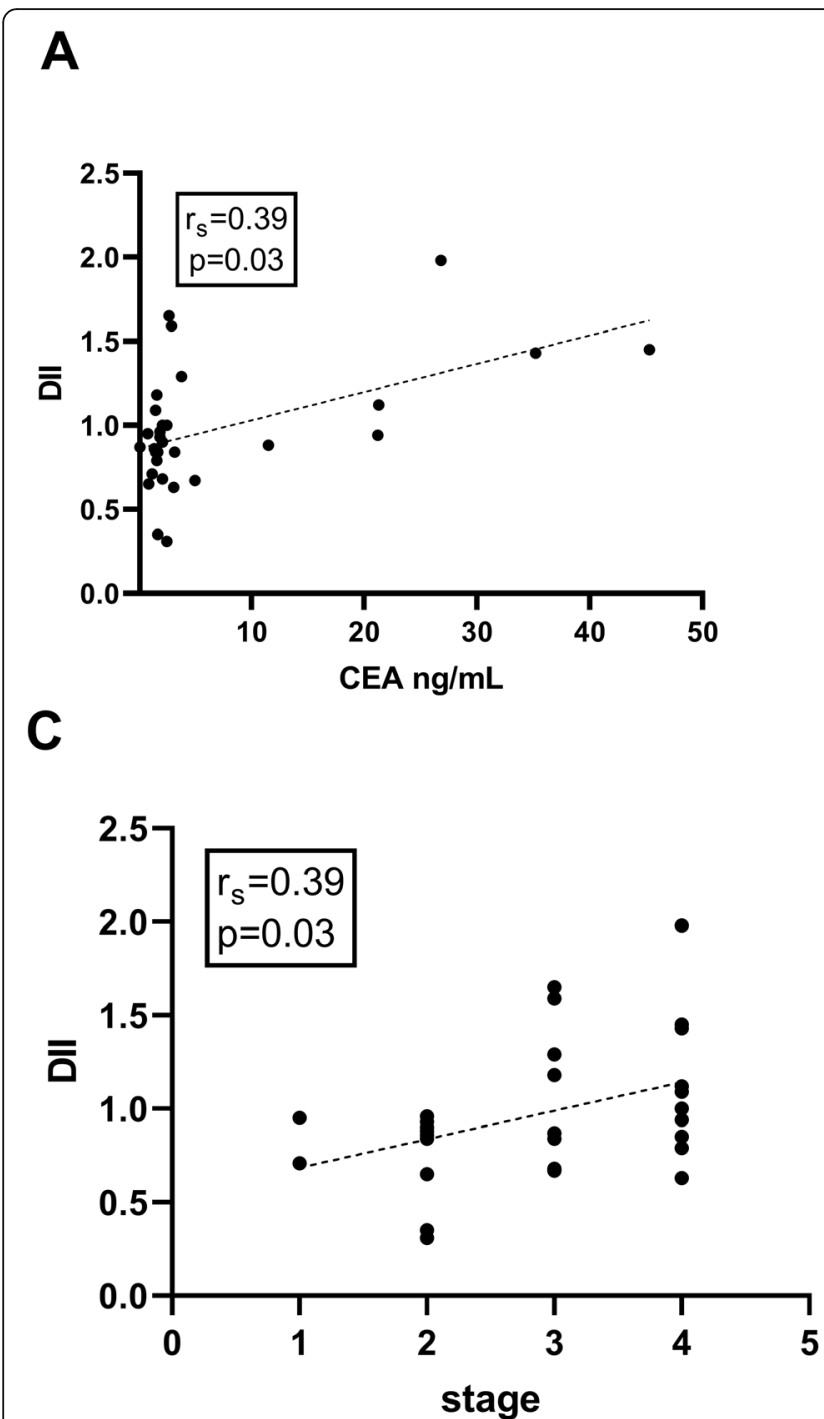

B

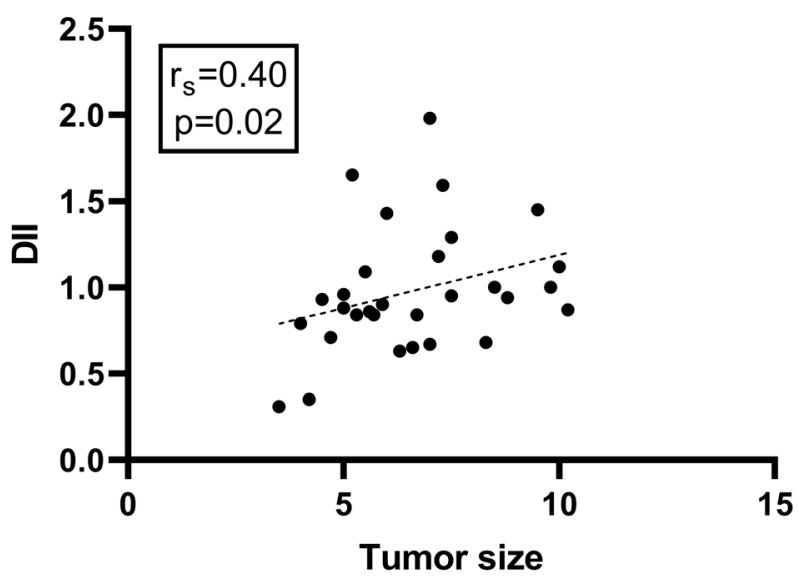

Fig. 4 DII in CRC patients correlated with CEA (a), tumor size (b), and tumor stage (c)

consistent with El-Gayar and his coworkers [13]. As for ALU 115 that represents absolute total DNA concentration, we found a significant increase in the level of absolute DNA concentration in CRC patients compared to healthy controls but non-significant difference was found between CRC patients and benign group. Similarly, El-Gayar et al. [13] and Hao et al. [14] found significantly higher levels of absolute DNA concentration in CRC group than healthy controls. However, in contrast to our findings, they found significantly higher levels of absolute DNA concentration in the CRC group compared to the benign group. This difference may be attributed to that previous studies were conducted on patients having poorly differentiated (grade III) tumors, with different histopathological states that might contribute to a significant increase in absolute cfDNA levels in CRC patients compared to the benign group. However, in our study, most patients had moderately differentiated (grade II) mucinous adenocarcinoma.

Additionally, we observed a significant increase in absolute DNA concentration in a benign group compared to the healthy control group. This finding came in agreement with Mead et al. [15] and contrasted by Bedin et al. [7] and El-Gayar et al. [13]. Our findings are supported by the fact that any benign disease condition may be accompanied by some sort of inflammation that may cause elevation of total DNA in our pathological control group. However, the discrepancy with Bedin et al. [7] and El-Gayar et al. [13] in this regard may be attributed to difference in sample sizes, and Bedin and his colleagues [7] used plasma as source of cfDNA, the exclusion of presence of inflammatory conditions in 


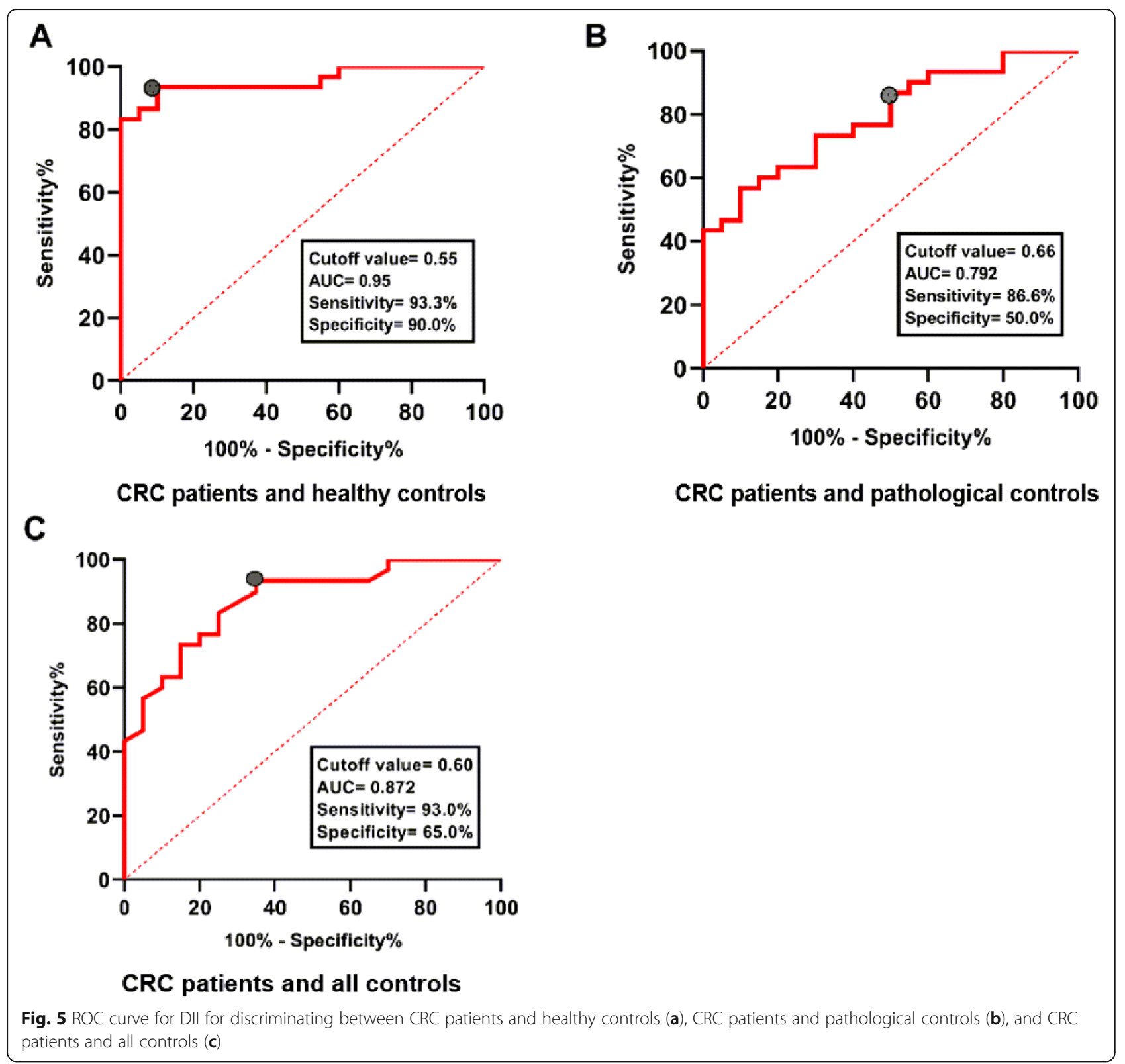

both benign and healthy group was done by full historytaking only, and there were no investigations done to confirm absence of hidden inflammatory conditions in our study and other studies.

As for ALU 247 concentration, which represents necrotic long DNA fragments, there was a significantly higher concentration of ALU 247 in CRC patients compared to both healthy controls and pathological controls. No statistically significant difference was found between pathological controls and healthy controls. This came in agreement with Mead et al. [15] who found a significant difference between CRC patients compared to healthy controls.
To assess the clinical relevance of our studied markers as early markers for CRC, there was a statistically significant difference in the levels of DII and ALU247 between patients with early stages without evidence of metastasis (stages I and II) and controls. However, non-significant difference was found between the two groups as regards CEA and ALU115. This came in accordance with Umetani et al. [12] and Arakawa et al. [16] who found significantly higher levels of DII in patients with early stages of CRC than in healthy volunteers.

To predict the prognostic significance of CEA, ALU 115,247 , and DII, we compared the previously mentioned markers with the status of metastasis; there were 
significantly higher levels of CEA and DII in patients with metastasis than those without metastasis. This suggests using both markers to differentiate between patients with early and late stages Also, a correlation study was done between the studied markers and both tumor size and stage. DII was found to have a significant positive correlation with tumor stage and tumor size while CEA was only found to have a significant positive correlation with tumor stage. Also, DII positively correlated with CEA. These findings came in agreement with El-Gayar et al. [13] and Arakawa et al. [16]. No significant difference was found between the metastatic and non-metastatic groups as regards ALU 115 and ALU 247. Similarly, El-Gayar et al. [13] found a nonsignificant difference in ALU 115 between the two groups.

\section{Conclusions}

Our study adds to previous research that suggests that DII is an early marker for CRC detection and may be used as a candidate biomarker for malignancy. Additionally, DII has prognostic significance as it correlated with tumor size and stage suggesting that it can be used to monitor disease progression and follow-up of the patients. Hence, this study highlights the utility of DII as a potential biomarker for CRC.

\section{Abbreviations}

ALU: Arthrobacter luteus; AUC: Area under the curve; CA 19.9: Carbohydrate antigen 19.9; CEA: Carcinoembryonic antigen; CfDNA: Cell-free DNA; CRC: Colorectal cancer; $C$ : Computerized tomography;

DNA: Deoxyribonucleic acid; DII: DNA integrity index; MRI: Magnetic resonance imaging; NPV: Negative predictive value; PCR: Polymerase chain reaction; PPV: Positive predictive value; ROC: Receiver-operating characteristic; TNM: $\mathrm{T}=$ tumor size, $\mathrm{N}=$ node involvement, and $\mathrm{M}=$ metastasis status

\section{Acknowledgements}

Not applicable

\section{Authors' contributions}

KS designed the data collection tools, monitored data collection for the whole research, interpreted the data, and revised the paper. MA collected the samples, collected patients' clinical data, and staged the patients. RS, RA, and $\mathrm{HH}$ carried out the laboratory work and analyzed it. RS and RA carried out statistical analysis. RS, RA, HH, and MA drafted the paper. All authors read and approved the final manuscript.

\section{Funding}

None.

\section{Availability of data and materials}

The datasets used and/or analyzed during the current study are available from the corresponding author on reasonable request.

\section{Ethics approval and consent to participate}

The study protocol was approved by the ethical committee of the Faculty of Medicine, Ain Shams University. Ethical approval reference number FMASU MD214/2018. Written informed consent was taken from all subjects before participating in this study.

\section{Consent for publication}

Not applicable

\section{Competing interests}

The authors declare that they have no competing interests.

\section{Author details}

${ }^{1}$ Clinical Pathology Department, Faculty of Medicine, Ain Shams University, Cairo, Egypt. ${ }^{2}$ General Surgery Department, Faculty of Medicine, Ain Shams University, Cairo, Egypt.

Received: 3 June 2020 Accepted: 10 July 2020

Published online: 22 September 2020

\section{References}

1. Bray F, Ferlay J, Soerjomataram I, Siegel RL, Torre LA, Jemal A (2018) Global cancer statistics 2018: GLOBOCAN estimates of incidence and mortality worldwide for 36 cancers in 185 countries. CA Cancer J Clin. 68:394-424

2. Bresalier RS, Kopetz S, Brenner DE (2015) Blood-based tests for colorectal cancer screening: do they threaten the survival of the fecal immunochemical test (FIT) test? Dig Dis Sci 60:664-671

3. Tóth K, Sipos F, Kalmár A, Patai AV, Wichmann B, Stoehr R et al (2012) Detection of methylated SEPT9 in plasma is a reliable screening method for both left- and right-sided colon cancers. PLoS One. 7(9):e46000

4. Wang X, Shi XQ, Zeng PW, Mo FM, Chen ZH (2018) Circulating cell free DNA as the diagnostic marker for colorectal cancer: a systematic review and meta-analysis. Oncotarget. 9:24514-24524

5. Duffy MJ, Lamerz R, Haglund C, Nicolini A, Kalousová M, Holubec L et al (2014) Tumor markers in colorectal cancer, gastric cancer and gastrointestinal stromal cancers: European group on tumor markers 2014 guidelines update. Int J Cancer. 134:2513-2522

6. Leszinski G, Lehner J, Gezer U, Holdenrieder S (2014) Increased DNA integrity in colorectal cancer. In Vivo 28:299-303

7. Bedin C, Enzo MV, Del Bianco P, Pucciarelli S, Nitti D, Agostini M (2017) Diagnostic and prognostic role of cell-free DNA testing for colorectal cancer patients. Int. J. Cancer. 140:1888-1898

8. Oliveira IBD, Hirata RDC (2018) Circulating cell-free DNA as a biomarker in the diagnosis and prognosis of colorectal cancer. Br J Pharmaceutical Sciences. 54:e17368

9. Ivancic MM, Megna BW, Sverchkov Y, Craven M, Reichelderfer M, Pickhardt PJ et al (2020) Noninvasive detection of colorectal carcinomas using serum protein biomarkers. J Surg Res. 246:160-169

10. Salvianti F, Pinzani P, Verderio P, Ciniselli CM, Massi D, De Giorgi V et al (2012) Multiparametric Analysis of Cell-Free DNA in Melanoma Patients. PLOS ONE. 7:e49843

11. Van Beers EH, Joosse SA, Ligtenberg MJ, Fles R, Hogervorst FB, Verhoef S et al (2006) A multiplex PCR predictor for aCGH success of FFPE samples. Br J Cancer 94:333-337

12. Umetani N, Kim J, Hiramatsu S, Reber HA, Hines OJ, Bilchik AJ et al (2006) Increased integrity of free circulating DNA in sera of patients with colorectal or periampullary cancer: direct quantitative PCR for ALU repeats. Clin. Chem. 52:1062-1069

13. El-Gayar D, El-Abd N, Hassan N, Ali R (2016) Increased free circulating DNA integrity index as a serum biomarker in patients with colorectal carcinoma. Asian Pac J Cancer Prev 17:939-944

14. Hao TB, Shi W, Shen XJ, Qi J, Wu XH, Wu Y et al (2014) Circulating cell-free DNA in serum as a biomarker for diagnosis and prognostic prediction of colorectal cancer. Br J Cancer. 111:1482-1489

15. Mead R, Duku M, Bhandari P, Cree IA (2011) Circulating tumor markers can define patients with normal colons, benign polyps, and cancers. Br. J. Cancer. 105:239-245

16. Arakawa S, Ozawa S, Ando T, Takeuchi H, Kitagawa Y, Kawase J et al (2019) Highly sensitive diagnostic method for colorectal cancer using the ratio of free DNA fragments in serum. Fujita Medical Journal. 5:14-20

\section{Publisher's Note}

Springer Nature remains neutral with regard to jurisdictional claims in published maps and institutional affiliations. 